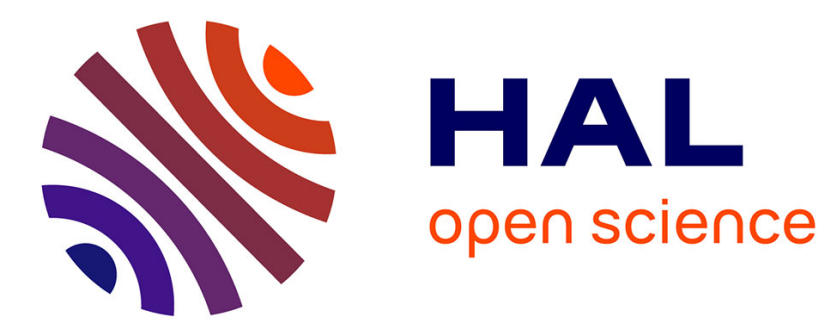

\title{
Perspective on long-lived nuclear spin states Jean-Nicolas Dumez
}

\section{To cite this version:}

Jean-Nicolas Dumez. Perspective on long-lived nuclear spin states. Molecular Physics, 2019, pp.1-11. 10.1080/00268976.2019.1644382 . hal-02348455

\section{HAL Id: hal-02348455 \\ https://hal.science/hal-02348455}

Submitted on 19 Jan 2021

HAL is a multi-disciplinary open access archive for the deposit and dissemination of scientific research documents, whether they are published or not. The documents may come from teaching and research institutions in France or abroad, or from public or private research centers.
L'archive ouverte pluridisciplinaire HAL, est destinée au dépôt et à la diffusion de documents scientifiques de niveau recherche, publiés ou non, émanant des établissements d'enseignement et de recherche français ou étrangers, des laboratoires publics ou privés. 


\title{
Perspective on long-lived nuclear spin states
}

\author{
Jean-Nicolas Dumez
}

CEISAM, CNRS UMR6230, Université de Nantes, 44300 Nantes, France

\section{ARTICLE HISTORY}

Compiled June 21, 2019

\begin{abstract}
This perspective article describes the concept of a long-lived nuclear spin state (LLS) and some of its applications. Classes of molecular environments for which nuclear spin order can be stored for extended periods of time are described, and applications to studies of molecular dynamics and interactions, diffusion NMR, and hyperpolarisation are presented. Some of the opportunities and challenges in the field are discussed.
\end{abstract}

\section{KEYWORDS}

Relaxation; molecular dynamics; hyperpolarisation; diffusion; microstructure

\section{Introduction}

Nuclear spin relaxation is of prime importance in nuclear magnetic resonance (NMR) spectroscopy and imaging (MRI). The relaxation properties of nuclear spins, including longitudinal and transverse relaxation rate constants, cross-relaxation rate constants, etc, provide a rich source of information on molecular structure and dynamics, as well as on microscopic environments. Relaxation times are also a measure of the 'memory' of a nuclear spin system. In some contexts, short relaxation times are beneficial in order to, e.g. run closely spaced consecutive scans without inter-scan interferences. In many cases, however, long relaxation times are desirable, to extend the timescales 
during which information may be stored with magnetic resonance. For an ensemble of uncoupled spins, the return to equilibrium of longitudinal magnetisation is the slowest relaxation process. As a result, the longitudinal relaxation time constant $T_{1}$ is often considered as the upper limit of the memory time of a spin system.

Long-lived nuclear spin order is a form of nuclear spin order that returns to equilibrium on a timescale that can be much longer that the longitudinal relaxation time constant $T_{1}$. A 'long-lived state' (LLS) is the corresponding operator, of which the longlived spin order is the expectation value. LLSs were first described in 2004 by Levitt and co-workers for a pair of spins $I=1 / 2[1,2]$. LLSs have the potential to extend the timescales that can be accessed in a range of experiments such as diffusion-ordered or exchange NMR spectroscopy, microstructure analysis, or ex-situ hyperpolarisation. Because their dependence on molecular dynamics and geometry is different from that of other relaxation parameters, LLS relaxation rate constants also provide a new class of NMR-based probes. All of these ideas have been explored over the past 15 years, and long-lived states have turned into an active area of research. The use of long-lived states in NMR spectroscopy and imaging presents many opportunities, such as novel approaches to ligand binding assays or hyperpolarised molecular imaging. Important challenges also remain for a move beyond proof-of-concept applications, such as the design or discovery of a molecule that simultaneously bears a LLS and is compatible with the process of interest.

In this perspective, the concept of long-lived states is first described, together with several generations of molecular structure that have been found or designed to bear long-lived states, and the corresponding NMR experiments. The use of LLS relaxation properties as a probe of structure and dynamics, and as a source of contrast is then described. Applications of LLS to store information on extended timescales are also detailed. Finally, some of the routes that are currently explored to expand the scope of LLS experiments are discussed. 


\section{What are long-lived states?}

\subsection{Definition and existence}

Long-lived nuclear spin order may be defined as a distribution of spin populations that returns to its equilibrium value on a timescale that is longer than that for the relaxation of longitudinal magnetisation (quantified by the longitudinal relaxation time constant $T_{1}$ ) [3]. It typically corresponds to population differences between manifolds of spin energy levels with different symmetry properties. The prototypical long-lived spin order concerns pairs of nuclear spins of spin quantum number $I=1 / 2$, and consists of the difference between the population of the singlet state

$$
\left|S_{0}\right\rangle=\frac{|\alpha \beta\rangle-|\beta \alpha\rangle}{\sqrt{2}}
$$

and the average population of the three triplet states:

$$
\begin{aligned}
\left|T_{+1}\right\rangle & =|\alpha \alpha\rangle, \\
\left|T_{0}\right\rangle & =\frac{|\alpha \beta\rangle+|\beta \alpha\rangle}{\sqrt{2}}, \\
\left|T_{-1}\right\rangle & =|\beta \beta\rangle .
\end{aligned}
$$

While the singlet state is antisymmetric with respect to permutation of the two spins, the triplet states are symmetric. This population difference is also called 'singlet order' or 'triplet-singlet imbalance'. The corresponding energy level diagram is shown in Fig. 1a for a pair of magnetically equivalent spins.

[Figure 1 about here.]

As is generally the case in NMR relaxation theory, the relaxation behaviour of LLSs is more conveniently described by first distinguishing the coherent spin interactions, that are identical for all the members of the ensemble, from the fluctuating spin interactions $[4,5,6]$. In high-resolution solution-state NMR, the former comprise the isotropic chemical shift and J coupling interactions, while the latter include the dipole-dipole, chemical shift anisotropy (CSA), quadrupolar and spin-rotation inter- 
actions.

Long-lived states exist when transitions under the effect of the fluctuating Hamiltonian are forbidden between manifolds of spin energy levels, or occur at a reduced rate. For example, singlet order for two spins 1 and 2 is not affected by the fluctuating intra-pair dipole dipole coupling $H_{12}^{D D}(t)$, because $H_{12}^{D D}$ is itself symmetric with respect to permutation of the two spins $[2,1,3]$. Since $H_{12}^{D D}(t)$ is the main relaxation mechanism for longitudinal magnetisation, the relaxation of singlet order can in comparison be greatly reduced. On the other hand, relaxation due to the dipole-dipole interaction with a third spin is attenuated for singlet order, not suppressed, to an extent that depends on geometry [4].

The relaxation properties of long-lived states have been thoroughly characterised $[7,8,10,11,12,4,13,3,5,6,14,15,16]$, with two main objectives. First, the understanding of LLS relaxation makes it possible to design molecules that are expected to display states that are exceptionally long-lived. This has recently led to the synthesis of a molecular scaffold with a central pair or ${ }^{13} \mathrm{C}$ spins, for which the singlet lifetime is longer than one hour [6]. In some cases, such as methyl groups [5], internal motion was found to play an important role in the properties of the long-lived state, and the observed LLS lifetime sometimes cannot be explained by considering a rigid molecule [17].

Second, establishing quantitative relations between LLS relaxation rate constants, and structural and dynamical properties, makes it possible to use LLS relaxation rate constants as new ways to probe the structure and dynamics of molecules $[8,13]$. Interestingly, because the relaxation mechanisms that are mainly responsible for $T_{1}$ are ineffective for long-lived states, the description of LLS relaxation requires accounting for interactions, such as the antisymmetric CSA or spin rotation [6], that are typically neglected in NMR relaxation studies.

Overall, the dependence of LLS relaxation properties on the geometry of spin interactions and molecular dynamics is complex, and in many cases population imbalances between spin-state manifolds of different symmetry return to their equilibrium value on a timescale that is shorter than that of longitudinal relaxation (i.e., $\left.T_{\mathrm{LLS}}<T_{1}\right)$. 


\subsection{Access and leakage}

The possibility to manipulate long-lived states experimentally is determined by coherent spin interactions. There are, however, conflicting requirements for the possibility to 'access' long-lived states using pulse sequences, and the degree of 'isolation' of these states at high magnetic fields. These conflicting requirements have led to several generations of LLS-bearing molecules or molecular fragments. The mathematical aspects of coherent manipulations of LLS in several regimes (strong chemical inequivalence, weak chemical inequivalence, chemical equivalence and magnetic inequivalence) and of the associated pulse sequences have been described by Pileio [9] and Levitt [3] and only the results will be summarised here.

The first demonstration of the existence of long-lived states involved a pair of ${ }^{1} \mathrm{H}$ spins with a difference in chemical-shift frequency offsets much larger than their mutual $\mathrm{J}$ coupling in the field of a high-resolution NMR magnet $[2,1]$. The corresponding energy level diagram is shown in Fig. 1b. In this case of large chemical inequivalence, while singlet order is still immune to the relaxation caused by the intra-pair dipoledipole interaction, the singlet and triplet states are not eigenstates of the coherent Hamiltonian. This property has the important consequence that free evolution under the effect of the coherent Hamiltonian at high field interconverts singlet order and other multi-spin terms. On the one hand, this provides a convenient route to generate singlet order. On the other hand, it also means that additional measures are required to lock the system in its singlet state: while singlet order may be long-lived, the other multi-spin terms into which it coherently evolves are not, and spin order is overall lost through fast relaxation of the latter [4]. Two solutions were proposed by Levitt and co-workers to prevent coherent evolution of singlet order. The first solution consists of applying a continuous radio-frequency pulse of sufficient intensity to suppress the coherent evolution [2]. This scheme makes it possible to carry out experiments entirely at high field, but it is demanding on hardware as it requires unusually long spin-locking field, of up to several minutes. The second solution consists of shuttling the sample from the high magnetic field region, where a precursor state is prepared, to a low magnetic field region, where the difference in chemical-shift frequency offsets is much smaller than the J coupling [1]. This also suppresses the coherent evolution of singlet 
order. This approach however requires a setup that makes it possible to move the sample out of the high field region sufficiently fast.

A breakthrough in the development of LLS was the introduction of pulse sequences to convert magnetisation into singlet order for nuclear spin pairs with differences in chemical-shift frequency offsets that are much smaller, at high field, than their mutual J coupling (so-called 'nearly chemically equivalent' spin pairs) [18]. The first, dubbed M2S (Magnetisation to Singlet), relies on trains of J-synchronised $180^{\circ}$ pulses to reintroduce singlet-triplet transitions [18]. While the method was first developed for audio-frequency pulse sequence for low field conversion, it also made it possible to prepare singlet order at high field for spin pairs that then do not require a spin-lock field or sample shuttling to be long-lived [19, 20]. Fig. 2 shows the corresponding pulse sequence diagram. Near-chemical-equivalence largely protects singlet order from coherent evolution into fast relaxing terms. While the application of a spin lock field can still extend the singlet lifetime, it does so only to a modest extent [19]. Other pulses sequences such as Spin-Lock Induced Crossing (SLIC) [21], based on a weak continuous irradiation, and frequency-swept versions $[22,23]$ were also introduced to convert magnetisation to singlet order for these systems. The M2S and SLIC sequences can also be used to prepare long-lived multi-spin states for pairs of chemically equivalent but magnetically inequivalent spins $[24,17,25,26]$

[Figure 2 about here.]

Long-lived states also exists for groups of nuclear spins that are both magnetically and chemically equivalent $[27,28,29,5,30]$. In this case, however, pulse sequences cannot be used to drive the conversion between magnetisation and LLS. A long-lived state may still be significantly populated with hyperpolarisation schemes such as dynamic nuclear polarisation $[27,29,30,31]$, or quantum-rotor induced polarisation [32, 28, 5]. While the LLS is NMR silent, it can be turned into population differences across observable transitions by a chemical reaction [27, 29], or by cross relaxation due to an external nuclear spins $[28,5,30,31]$. These preparation and conversion mechanisms have been demonstrated experimentally, but they provide less control than coherent manipulations and have been less exploited so far. 


\section{Probing molecular properties}

The measurement of relaxation rate constants by NMR spectroscopy provides access to quantitative information on the structure and dynamics of molecules in solution. For the analysis of complex solution mixtures and semi-solid samples, and for in vivo localised spectroscopy, relaxation mechanisms are used as a source of contrast to separate molecules of interest from background signals. Different relaxation rate constants encode different properties, and for this reason the relaxation properties of long-lived nuclear spin states can provide a new window on structure and dynamics, and provide new types of filters. Proof-of-principle experiments have been reported in all of these areas.

Singlet relaxation has been used as a probe of the interaction between smallmolecule ligands and proteins $[33,34,35]$. These studies used singlet states of the ligand, formed by pairs of chemically inequivalent spins, that require continuous RFbased spin locking to be long-lived at high field. Salvi et al. showed that the LLS relaxation time constant $T_{\text {LLS }}$ provides very good contrast between the free and bound form of the ligand, because small changes in chemical shifts, such as those induced by the ligand-protein interaction, have a significant effect on the efficiency with which spin locking prevents LLS leakage [33]. This contrast was used to detect weakly binding ligands in a fragment-based drug design approach. Since not all possible ligands have a suitable spin pair to form a long-lived state, ligand binding analyses were carried out using competition assay, or LLS-bearing molecular tags [34]. Fig. 3 shows the example identification of a weak binder in a mixture using a competition assay.

[Figure 3 about here.]

Singlet states have also been used in methods to retrieve the signals of selected molecules from a complex background. DeVience et al. described a pulse sequence to select signals from a specific singlet-forming pair of coupled spins, such as geminal protons in N-aspartic acid (NAA) and suppress other proton signals. Singlet order is prepared with sequence of pulses, sustained with a continuous spin lock, and converted back to magnetisation [36]. Selectivity with respect to ${ }^{1} \mathrm{H}$ spins that do not form singlet states is obtained because terms other than singlet-order are saturated during the spin 
lock, and also filtered out by phase cycling. Selectivity among different singlet-forming spin pairs is achieved by choosing the parameters of the conversion pulse sequence to be more effective for the spin pair of interest. An improved implementation of this concept was described by Ivanov and co-workers, using a magnetisation to singlet conversion pulse sequence based on an adiabatic sweep, which led to an improved contrast between the selected singlet-forming ${ }^{1} \mathrm{H}$ pair and the suppressed background $[37,38]$. Singh et al. have proposed to use singlet relaxation as a tool for spectral editing in mixtures [39].

The dependence of singlet relaxation rate constants on geometry has been exploited to probe the relative orientation of CSA tensors as well as torsional angles in a small molecules $[8,40]$. The relaxation time constant $T_{S}$ of singlet order for a pair of spins 1 and 2 caused by an interaction $H^{\lambda}(t)$ depends on the degree of correlation between the fluctuations of $H_{1}^{\lambda}$ and $H_{2}^{\lambda}$. For example, relaxation due to the CSA interaction depends on the angle between the two CSA tensors $\sigma_{1}^{\text {aniso }}$ and $\sigma_{2}^{\text {aniso }}$. This property was used by Ahuja et al. to probe the relative orientation of ${ }^{1} \mathrm{H}$ CSA tensors in substituted benzene rings [8]. On the other hand, the contribution to $T_{S}$ caused by the dipole-dipole interaction with a third spin 3 depends on the angles between the internuclear vector $\vec{r}_{13}$ and $\vec{r}_{23}$. By measuring $T_{S}$ for a spin pair whose singlet relaxation is dominated by a single neighbouring proton, and assuming fixed bond length and angles, Tayler et al. showed that torsional angle around the $\mathrm{C}^{\beta} / \mathrm{C}^{\gamma}$ bond could be measured for a phenylalanine derivative [40].

\section{Accessing longer timescales}

For experiments that consist of multiple consecutive scans, be it for signal averaging, additional dimensions or monitoring, the relaxation rate constant of longitudinal magnetisation is often found to be longer than what the experimenter would wish. This results in inter-scan delays that account for most of the experiments' duration, and drives the design of an array of strategies to effectively increase relaxation rate constants. On the other hand, there exists a vast range of NMR experiments where longitudinal magnetisation is used for storage. This includes storage of the labelling 
of the spins' position or chemical-shift in the form of an amplitude modulation, and storage of enhanced spin order prepared by hyperpolarisation methods. In these cases, longitudinal relaxation sets the upper limit for the timescale over which information may be encoded. By using instead long-lived nuclear spin states for storage, longer timescales may in principle be accessed.

\subsection{Storage of position and chemical-shift information}

Using magnetic-field gradients, information about the spins' position in space can be encoded as a phase or amplitude modulation. In diffusion NMR experiments, position encoding and decoding steps are separated by a delay, and changes in the spins' position during the delay results in an attenuation that can be modelled to estimate translational diffusion coefficients. For free diffusion, the minimum spatial scale of translational diffusion that can be characterised is determined by the maximum gradient area that can be achieved, and by the duration of the diffusion delay. While the former is dictated by hardware limitations, the latter is, in classic diffusion experiments, limited by longitudinal relaxation. Using singlet order instead of longitudinal magnetisation for storage, Bodenhausen and co-workers have shown that longer diffusion delays could be used in diffusion NMR experiments [41]. As a result, diffusion coefficients were measured for a biomolecule, ubiquitin, using a reduced range of gradient areas [42].

Long-lived states have also been used in diffusion NMR experiments designed to characterise the microstructure of materials. Such experiments require storage delays that are sufficiently long for molecules to experience a restricted translational diffusion. For example, for a molecule with a given diffusion coefficient, probing the size of larger pores requires longer diffusion delays. Price and co-workers have shown, using the singlet state formed by a proton pair in 2,3-dibromothiophene, that longer diffusion delays could be used, in order to probe the size of larger pores [43, 44]. Pileio and coworkers further increased the accessible spatial scale by using singlet states for pairs of nearly-equivalent spins, with lifetimes of several minutes, to probe pores of up to 2 $\mathrm{mm}$ [45]. The diffusion-encoding gradients were embedded in the M2S pulse sequence. They also showed that 'singlet-assisted diffusion' experiments also help to characterise 
higher-order microstructural features such as tortuosity [46].

An alternative approach to encode position information is to use spatially selective manipulations of the spins. With this objective, Pileio and co-workers have designed a spatially selective version of the M2S and S2M pulse sequences, in which hard pulses are replaced by combinations of soft pulses and magnetic-field gradient. The selS2M sequence was used in phantom experiments to label a slice of a solutions of molecules with pairs of nearly equivalent ${ }^{1} \mathrm{H}$ or ${ }^{13} \mathrm{C}$ spins, and monitor either diffusion or flow in direct rather than reciprocal space, as illustrated in Fig. 4 [47].

[Figure 4 about here.]

In two-dimensional correlation experiments, it is the spins' chemical-shift in the indirect dimension that is encoded as a phase or amplitude modulation. In experiments for the characterisation of chemical exchange (EXSY) and cross relaxation (NOESY), this information is stored as longitudinal magnetisation, and this sets a limit to the duration of the relaxation or exchange delay. In EXSY, this in turns determines the range of exchange rate constants that can be measured accurately, with slower exchange processes requiring longer delays. Bodenhausen and co-workers have shown that the encoding of chemical sites could instead be stored with singlet order, using modified EXSY pulse sequences [48]. This required pulse sequence elements for the conversion from magnetisation to singlet order that are broadband with respect to sum and difference chemical-shift for the spin pairs and to their $\mathrm{J}$ coupling. The SS-EXSY was used to monitor slow conformational exchange in a partly deuterated saccharide.

\subsection{Storage of hyperpolarised spin order}

The maximum timescale during which spin order can be stored is a particularly important concern in the field of hyperpolarised magnetic resonance. Hyperpolarisation consists of increasing population differences between nuclear spin energy levels much beyond their thermal equilibrium values, in order to increase the sensitivity of NMR experiments. Currently, for solution-state and in vivo NMR, the most commonly used hyperpolarisation schemes involve an irreversible step: enhanced spin order is prepared 
only once and subsequently decays. When hyperpolarised spin order is kept in the form of longitudinal magnetisation, longitudinal relaxation again sets an upper limit on the time scale during which this order, and the corresponding signal, remains available. This limitation is particularly important for applications of hyperpolarisation to the monitoring of time-dependent processes. Long-lived states have the potential to increase significantly the timescales accessible in hyperpolarised magnetic resonance, and this was recognised already when their existence was first reported by Levitt and co-workers [1].

\subsubsection{Dissolution dynamic nuclear polarisation}

In dissolution dynamic nuclear polarisation (D-DNP), hyperpolarised substrates are first prepared by DNP in the solid state, then rapidly dissolved with a hot solvent and transferred to a solution-state NMR spectrometer or an MRI scanner for detection [49]. For the DNP step, the most common approach consists of: i/ mixing the substrates and radical-bearing molecules in a glass-forming solvent, ii/ placing the sample at a temperature of 1.1 to $4.2 \mathrm{~K}$ and a magnetic field of 3 to $10 \mathrm{~T}$; iii/ irradiating the sample with microwave to build up nuclear polarisation $[50,51]$. While the combined effects of DNP and a temperature jump can result in signal enhancements of over $10^{4}$, the enhanced spin order decays irreversibly after dissolution and the timescales that can be probed with DNP-hyperpolarised substrates are limited by longitudinal relaxation .

The possibility to store DNP-enhanced spin order using long-lived nuclear spin states was first demonstrated by Bodenhausen and co-workers, who transferred DNPenhanced ${ }^{13} \mathrm{C}$ magnetisation to ${ }^{1} \mathrm{H}$ singlet order in the dipeptide Ala-Gly at high field, where the LLS was sustained with an RF spin lock [52]. The experiment was later improved by directly preparing ${ }^{1} \mathrm{H}$ magnetisation with DNP, and shuttling the sample in a low field region during the storage delay, using the ${ }^{1} \mathrm{H}$ pair of 3-bromothiophene2-carboxylic acid [53,54]. As explained in section 2.2, singlet order can be preserved for extended periods of time at high magnetic fields with no need for an RF spin lock in the case of pairs of nearly chemically equivalent spins. Pileio et al. have shown using the M2S/S2M pulse sequences for back-and-forth conversion between magnetisation 
and singlet order, that DNP-enhanced spin order could be stored for over 30 min using pairs of nearly equivalent ${ }^{13} \mathrm{C}$ spins [55]. They also demonstrated the acquisition of MR images on a pre-clinical scanner.

A possible route to prepare hyperpolarised singlet order, used in the examples described so far, consists of preparing enhanced magnetisation (single-spin longitudinal order) with D-DNP, then converting it to singlet order using a pulse sequence in the high-field NMR or MRI system. An alternative, more direct route consists of exploiting the fact that when very high polarisation levels are achieved by DNP, an appreciable level of singlet order is also generated (the level of singlet order is given by $-p^{2} / 3$, where $p$ is the polarisation level). This was first shown by Tayler et al., with doubly ${ }^{13} \mathrm{C}-$ labelled pyruvic acid [56]. For this system there exists a large chemical-shift difference between the two ${ }^{13} \mathrm{C}$ spins, and the use of direct singlet preparation scheme makes it possible to avoid the need for pulse-sequence based conversion at high-field, where an $\mathrm{RF}$ irradiation would then be necessary to sustain the long-lived state.

The direct enhancement of singlet order is also possible for pairs of nearly chemically equivalent spins, as shown by Elliott et al. , who used D-DNP to prepare hyperpolarised singlet order in monodeuterated methyl groups, selected to have a chemicalshift difference between the two protons of the methyl group that is simultaneously much smaller than the intra-pair $\mathrm{J}$ coupling, and large enough to be able to drive the conversion between magnetisation and singlet order with an RF pulse sequence [57]. As already referred to in section 2.2, the direct hyperpolarisation of long-lived states is also particularly useful in the case of systems of equivalent spins, for which no pulse-sequence based conversion is possible [29, 30, 31]. This is illustrated in Fig. 5 with the DNP-based preparation of a long-lived state in methyl groups. In this case, A ${ }^{1} \mathrm{H}$ long-lived state is populated by DNP in the solid state, and yields population differences across observable transitions (for both ${ }^{1} \mathrm{H}$ and ${ }^{13} \mathrm{C}$ ) by cross-relaxation in solution.

[Figure 5 about here.] 


\subsubsection{Parahydrogen-based hyperpolarisation}

Hyperpolarised spin order can be prepared efficiently and with comparatively simple instrumentation for dihydrogen gas. Dihydrogen exists as two spin isomers, ortho and para, corresponding to the three symmetric (triplet) states and the antisymmetric (singlet) state of the spin pair. The existence of spin isomers is due to the Pauli principle and is only observed for small and highly symmetric molecules. For dihydrogen, the ortho and para forms interconvert efficiently when the gas is in contact with a paramagnetic solid, but an out-of-equilibrium ortho/para distribution can otherwise be retained for several days. At room temperature, the four energy levels are nearly equally populated, resulting in a 75:25 ortho:para distribution. 'Para-hydrogen gas' (dihydrogen gas with a non equilibrium ortho:para distribution) is prepared by flowing dihydrogen gas at temperatures of 20 to $80 \mathrm{~K}$, in the presence of a paramagnetic solid particles. Parahydrogen may be seen as a reservoir of long-lived singlet order. While parahydrogen is NMR silent and is unaffected by pulse sequences, its spin order can be released by a chemical addition. The addition is either directly onto a substrate of interest [58] (the PHIP mechanism, parahydrogen induced polarisation), in which case the enhanced spin order on the substrate is not renewable, or onto a metal centre on which a substrate is bound reversibly, in which case through-bond spin order transfer to the substrate spins can occur multiple times over several complex formations [59] (the SABRE mechanism, signal amplification by reversible exchange).

The connection between PHIP and long-lived states was illustrated experimentally in 2006 by Jonischkeit et al., who measured effective relaxation times at high and low field for the hydrogenation of symmetrically and asymmetrically substituted alkynes [60]. Several other asymmetrically substituted alkynes and alkenes have been hydrogenated with parahydrogen to prepare singlet states that are long-lived at low field. In particular, the hydrogenation of propene into hyperpolarised propane gas could potentially be used for low-field MRI of the lungs [61]. The hydrogenation of symmetric molecules, such that the two protons from parahydrogen remain chemically equivalent in the product molecule, has the advantage that singlet order for the ${ }^{1} \mathrm{H}$ pair remains long-lived at high magnetic field after the chemical addition, as illustrated by Franzoni et al. [62]. In that case, several strategies have been reported to generate 
observable signals from the parahydrogen-derived singlet order. These include, in the case of magnetically inequivalent spins, the conversion from singlet order to magnetisation for the ${ }^{1} \mathrm{H}$ pair, with pulse sequences that exploit difference in $\mathrm{J}$ coupling with other spins $[63,64]$. Jerschow and co-workers have also shown that a chemical reaction could be used to lift the chemical equivalence of the two protons [65]. Interestingly, pulse sequences for the conversion from singlet order to homonuclear magnetisation for pairs of nearly-chemically-equivalent spins can also be used, with little to no modification, to convert singlet order to heteronuclear magnetisation, in cases when the difference in $\mathrm{J}$ couplings with the heteronucleus is much smaller than the intra-pair coupling [66, 67]. This was used to hyperpolarise ${ }^{13} \mathrm{C}$ spins at high field after addition of parahydrogen. While the release of singlet order by a chemical addition and its conversion to observable magnetisation predate the introduction of the concept of long-lived states, the LLS methodology has increased the range of chemical systems that can be addressed, with efficient conversion sequences for pairs near-equivalent spins, as well as sequences to prepare ${ }^{13} \mathrm{C}$ magnetisation from ${ }^{1} \mathrm{H}$ singlet order.

Long-lived states have also been used to store hyperpolarised spin order derived from parahydrogen with the SABRE approach. Also in this case, singlet order can be prepared from SABRE enhanced magnetisation with an RF pulse sequence at high field, as shown by Roy et al. $[68,69]$. They used pairs of chemically inequivalent spins in, e.g., nicotinamide [68], and pairs of chemically nearly equivalent spins, in pyridazine derivatives [69]. With partial deuteration of the substrate, the resulting hyperpolarised long-lived spin orders can be stored for several minutes at low field. The SABRE process can also be exploited to generate singlet order directly for pairs of heteronuclear spins. This was demonstrated by Theis et al., with molecules that bear doubly ${ }^{15} \mathrm{~N}$ labelled diazirine tags [70]. They showed that in this case the polarisation transfer condition is field independent as long as the nitrogens are chemically equivalent, and that long-lived singlet order for the ${ }^{15} \mathrm{~N}$ pair can have a lifetime of over 20 min at 3 G. Since the two nitrogen are chemically inequivalent at high field, an enhanced anti phase doublet is obtained upon $90^{\circ}$ excitation after transfer to the detection magnet. Fig. 6 shows the result of a SABRE experiment on a ${ }^{15} \mathrm{~N}_{2}$-diazirine. Note that while the SABRE process, in contrast to PHIP and D-DNP, can be implemented 
as a multi-shot experiment, storage of SABRE-enhanced spin order is still relevant for a variety of proposed applications such as in vivo molecular imaging, which would require separating the hyperpolarised substrate from the SABRE catalyst.

[Figure 6 about here.]

\section{Discussion}

Long-lived nuclear spin states were first described in 2004 [1]. In the original publication of Levitt and co-workers, applications to the storage of spin order for flow, diffusion and hyperpolarisation experiments were already envisioned. Several proof-ofconcepts experiments have now been demonstrated in all of these areas. In parallel, the LLS theory and methodology have expanded and led to a much broader scope of chemical systems and experimental conditions for which timescales may be extended using long-lived states. The translation of these developments to the applications proposed so far in (bio)chemistry and biomedicine will depend on the possibility to find or design compounds that combine: i/ a chemical structure that is compatible with the chemical environment and/or reaction of interest, and, ii/ a long-lived state with a significantly enhanced lifetime in realistic experimental conditions.

Several routes are being explored, which may lead to molecules that would be used beyond proof-of-concept experiments. The longest LLS relaxation time constants so far have been measured for pairs of nearly chemically equivalent spins, in molecules designed such that known mechanisms for LLS relaxation would be minimised. These 'designer molecules', such as doubly ${ }^{13} \mathrm{C}$-labelled oxalates with $T_{S}$ values of more than 1 hour [14], are in addition chosen to be easily functionalised, and may thus be further modified to be water-soluble, or to achieve molecular sensing. A remaining challenge for these molecules is to design molecular scaffolds that also minimise the contribution of paramagnetic dissolved dioxygen to LLS relaxation.

Conversely, a variety of chemical moieties or molecular fragments have been identified as potential molecular tags, that can be attached to a molecule of interest $[35,71,70]$. This approach has however so far involved pairs of spins with a difference in chemical shift that is too large for singlet order to be long-lived at high magnetic 
field in the absence of RF spin locking. While this was used as an advantage to probe protein ligand interactions [35], it is a limitation when LLS are used to store spin order, since long RF irradiation may not always be possible because of hardware- or sample-related limitations. For these systems, the possibility to prepare singlet order directly by hyperpolarisation methods, based on either DNP or parahydrogen $[60,56]$, is particularly useful, as it circumvents the high-field RF-pulse-based conversion from magnetisation to singlet order. Molecular tags with pairs of near equivalent spins would be particularly useful to explore in vivo applications of long-lived states.

Hyperpolarised spin order in systems of magnetically equivalent spins, such as $\mathrm{CH}_{2}$ groups or methyl groups [30,28], also has the advantage that it can be prepared in the vast number of molecules that include these chemical moieties. However, the cross-relaxation process that is needed to generate population differences across observable transitions from the silent LLS provides little control, and also limits the lifetime that may be achieved. In addition, fast internal motion or a highly anisotropic overall tumbling is then required for the LLS to relax significantly more slowly than longitudinal magnetisation [31].

A general challenge for the use of long-lived states to store hyperpolarise singlet order is the fact that the LLS concept intrinsically involves multiple spins. A pair of spins $I=1 / 2$ is needed to form a singlet state, and, in the case of chemically equivalent spins, neighbouring spins are needed to lift the magnetic equivalence. For some compounds, while the singlet order of a ${ }^{1} \mathrm{H}$ or ${ }^{13} \mathrm{C}$ spin pair may be significantly longer lived than the longitudinal magnetisation of the same spin system, the increase in lifetime can be less dramatic when compared to the $T_{1}$ value of a singly- ${ }^{13} \mathrm{C}$-labelled compound [72]. Also, molecular platforms have been described in which nitrogen-15 spins have $T_{1}$ values of more than $10 \mathrm{~min}$ at high field [73].

Perhaps the most directly useful case concerns compounds that happen to have pairs of chemically equivalent and a small degree of magnetic inequivalence. For such systems singlet order can be efficiently converted to and from magnetisation -which can be heteronuclear magnetisation, and no RF spin lock is needed for singlet order to be long-lived at high field. Examples are $\left[1-{ }^{13} \mathrm{C}\right]$ fumarate and dimethylmaleate, which can be hyperpolarised with the PHIP method $[62,67]$. 
For applications that use $T_{S}$ measurements as a probe of structure, dynamics and interactions, the challenge is also to find a suitable spin system to prepare singletorder. From that point of view, the use of competitive binding assays is an interesting opportunity, as it relaxes the requirement to finding just one ligand with a long-lived state.

In summary, the initial idea of "extending the memory of nuclear spins [...] well beyond the $T_{1}$ limit" has led to a wealth of new concepts in nuclear spin relaxation, and applications in NMR spectroscopy and imaging. Recent development in the field show that there is steady progress towards the design of molecules that will unlock the potential of long-lived nuclear spin states.

\section{Acknowledgement(s)}

I am very grateful to many colleagues for discussions on long-lived nuclear spin states.

\section{References}

\section{References}

[1] M. Carravetta, O.G. Johannessen and M.H. Levitt, Phys. Rev. Lett. 92 (15), 1-4 (2004).

[2] M. Carravetta and M.H. Levitt, J. Am. Chem. Soc. 126 (20), 6228-6229 (2004).

[3] M.H. Levitt, Annu. Rev. Phys. Chem. 63 (1), 89-105 (2012).

[4] G. Pileio, Prog. Nucl. Magn. Reson. Spectrosc. 56 (3), 217-231 (2010).

[5] J.N. Dumez, P. Håkansson, S. Mamone, B. Meier, G. Stevanato, J.T. Hill-Cousins, S.S. Roy, R.C. Brown, G. Pileio and M.H. Levitt, J. Chem. Phys. 142 (4), 044506 (2015).

[6] G. Stevanato, S.S. Roy, J. Hill-Cousins, I. Kuprov, L.J. Brown, R.C. Brown, G. Pileio and M.H. Levitt, Phys. Chem. Chem. Phys. 17 (8), 5913-5922 (2015).

[7] M. Carravetta and M.H. Levitt, J. Chem. Phys. 122 (21), 214505 (2005).

[8] P. Ahuja, R. Sarkar, P.R. Vasos and G. Bodenhausen, J. Chem. Phys. 127 (13), $134112(2007)$. 
[9] G. Pileio, Prog. Nucl. Magn. Reson. Spectrosc. 98-99, 1-19 (2017).

[10] G. Pileio and M.H. Levitt, J. Magn. Reson. 187 (1), 141-145 (2007).

[11] G. Pileio and M.H. Levitt, J. Chem. Phys. 130 (21), 214501 (2009).

[12] M.H. Levitt, in Encycl. Magn. Reson. (John Wiley \& Sons, Ltd, Chichester, UK, 2010).

[13] M.C. Tayler and M.H. Levitt, Phys. Chem. Chem. Phys. 13 (20), 9128-9130 (2011).

[14] G. Stevanato, J.T. Hill-Cousins, S.S. Roy, L.J. Brown, R.C. Brown, G. Pileio and M.H. Levitt, Angew. Chemie - Int. Ed. 54 (12), 3740-3743 (2015).

[15] S.J. Elliott, L.J. Brown, J.N. Dumez and M.H. Levitt, Phys. Chem. Chem. Phys. 18 (27), 17965-17972 (2016).

[16] S.J. Elliott, L.J. Brown, J.N. Dumez and M.H. Levitt, J. Magn. Reson. 272, 87-90 (2016).

[17] Y. Feng, T. Theis, X. Liang, Q. Wang, P. Zhou and W.S. Warren, J. Am. Chem. Soc. 135 (26), 9632-9635 (2013).

[18] G. Pileio, M. Carravetta and M.H. Levitt, Proc. Natl. Acad. Sci. 107 (40), 1713517139 (2010).

[19] M.C. Tayler and M.H. Levitt, Phys. Chem. Chem. Phys. 13 (13), 5556 (2011).

[20] G. Pileio, J.T. Hill-Cousins, S. Mitchell, I. Kuprov, L.J. Brown, R.C.D. Brown and M.H. Levitt, J. Am. Chem. Soc. 134 (42), 17494-17497 (2012).

[21] S.J. Devience, R.L. Walsworth and M.S. Rosen, Phys. Rev. Lett. 111 (17), 1-5 (2013).

[22] T. Theis, Y. Feng, T. Wu and W.S. Warren, J. Chem. Phys. 140 (1), 014201 (2014).

[23] A.N. Pravdivtsev, A.S. Kiryutin, A.V. Yurkovskaya, H.M. Vieth and K.L. Ivanov, J. Magn. Reson. 273, 56-64 (2016).

[24] Y. Feng, R.M. Davis and W.S. Warren, Nat. Phys. 8 (11), 831-837 (2012).

[25] Y. Feng, T. Theis, T.L. Wu, K. Claytor and W.S. Warren, J. Chem. Phys. 141 (13), 134307 (2014).

[26] K. Claytor, T. Theis, Y. Feng, J. Yu, D. Gooden and W.S. Warren, J. Am. Chem. Soc. 136 (43), 15118-15121 (2014). 
[27] W.S. Warren, E. Jenista, R.T. Branca and X. Chen, Science (80-. ). 323 (5922), 1711-1714 (2009).

[28] B. Meier, J.N. Dumez, G. Stevanato, J.T. Hill-Cousins, S.S. Roy, P. Håkansson, S. Mamone, R.C.D. Brown, G. Pileio and M.H. Levitt, J. Am. Chem. Soc. 135 (50), 18746-9 (2013).

[29] A. Bornet, X. Ji, D. Mammoli, B. Vuichoud, J. Milani, G. Bodenhausen and S. Jannin, Chem. - A Eur. J. 20 (51), 17113-17118 (2014).

[30] D. Mammoli, B. Vuichoud, A. Bornet, J. Milani, J.N. Dumez, S. Jannin and G. Bodenhausen, J. Phys. Chem. B 119 (10), 4048-4052 (2015).

[31] J.N. Dumez, B. Vuichoud, D. Mammoli, A. Bornet, A.C. Pinon, G. Stevanato, B. Meier, G. Bodenhausen, S. Jannin and M.H. Levitt, J. Phys. Chem. Lett. 8 (15), 3549-3555 (2017).

[32] M. Icker and S. Berger, J. Magn. Reson. 219, 1-3 (2012).

[33] N. Salvi, R. Buratto, S. Ulzega, I.R. Rebollo, A. Angelini, C. Heinis and G. Bodenhausen, J. Am. Chem. Soc. 134 (3), 11076-11079 (2012).

[34] R. Buratto, D. Mammoli, E. Chiarparin, G. Williams and G. Bodenhausen, Angew. Chemie - Int. Ed. 53 (42), 11376-11380 (2014).

[35] R. Buratto, A. Bornet, J. Milani, D. Mammoli, B. Vuichoud, N. Salvi, M. Singh, A. Laguerre, S. Passemard, S. Gerber-Lemaire, S. Jannin and G. Bodenhausen, ChemMedChem 9 (11), 2509-2515 (2014).

[36] S.J. Devience, R.L. Walsworth and M.S. Rosen, NMR Biomed. 26 (10), 1204-1212 (2013).

[37] A.S. Kiryutin, H. Zimmermann, A.V. Yurkovskaya, H.M. Vieth and K.L. Ivanov, J. Magn. Reson. 261, 64-72 (2015).

[38] A.S. Kiryutin, A.N. Pravdivtsev, A.V. Yurkovskaya, H.M. Vieth and K.L. Ivanov, J. Phys. Chem. B 120 (46), 11978-11986 (2016).

[39] M. Singh, V. Kumar Soni, R. Mishra and N.D. Kurur, Anal. Chem. 88 (6), $3004-$ 3008 (2016).

[40] M.C.D. Tayler, S. Marie, A. Ganesan and M.H. Levitt, J. Am. Chem. Soc. 132 (24), 8225-8227 (2010).

[41] S. Cavadini, J. Dittmer, S. Antonijevic and G. Bodenhausen, J. Am. Chem. Soc. 
127 (45), 15744-15748 (2005).

[42] P. Ahuja, R. Sarkar, P.R. Vasos and G. Bodenhausen, J. Am. Chem. Soc. 131 (22), 7498-7499 (2009).

[43] N.N. Yadav, A.M. Torres and W.S. Price, J. Magn. Reson. 204 (2), 346-348 (2010).

[44] A.M. Torres, B. Ghadirian and W.S. Price, RSC Adv. 2 (8), 3352-3360 (2012).

[45] G. Pileio and S. Ostrowska, J. Magn. Reson. 285, 1-7 (2017).

[46] M.C. Tourell, I.A. Pop, L.J. Brown, R.C. Brown and G. Pileio, Phys. Chem. Chem. Phys. 20 (20), 13705-13713 (2018).

[47] G. Pileio, J.N. Dumez, I.A. Pop, J.T. Hill-Cousins and R.C. Brown, J. Magn. Reson. 252, 130-134 (2015).

[48] R. Sarkar, P.R. Vasos and G. Bodenhausen, J. Am. Chem. Soc. 129 (2), 328-334 (2007).

[49] J.H. Ardenkjaer-Larsen, B. Fridlund, A. Gram, G. Hansson, L. Hansson, M.H. Lerche, R. Servin, M. Thaning and K. Golman, Proc. Natl. Acad. Sci. 100 (18), 10158-10163 (2003).

[50] A. Bornet and S. Jannin, J. Magn. Reson. 264, 13-21 (2016).

[51] B. Plainchont, P. Berruyer, J.N. Dumez, S. Jannin and P. Giraudeau, Anal. Chem. 90 (6), 3639-3650 (2018).

[52] P.R. Vasos, A. Comment, R. Sarkar, P. Ahuja, S. Jannin, J.P. Ansermet, J.A. Konter, P. Hautle, B. van den Brandt and G. Bodenhausen, Proc. Natl. Acad. Sci. 106 (44), 18469-18473 (2009).

[53] A. Bornet, S. Jannin and G. Bodenhausen, Chem. Phys. Lett. 512 (4-6), 151-154 (2011).

[54] P. Ahuja, R. Sarkar, S. Jannin, P.R. Vasos and G. Bodenhausen, Chem. Commun. 46 (43), 8192-8194 (2010).

[55] G. Pileio, S. Bowen, C. Laustsen, M.C.D. Tayler, J.T. Hill-Cousins, L.J. Brown, R.C.D. Brown, J.H. Ardenkjaer-Larsen and M.H. Levitt, J. Am. Chem. Soc. 135 (13), 5084-5088 (2013).

[56] M.C. Tayler, I. Marco-Rius, M.I. Kettunen, K.M. Brindle, M.H. Levitt and G. Pileio, J. Am. Chem. Soc. 134 (18), 7668-7671 (2012). 
[57] S.J. Elliott, B. Meier, B. Vuichoud, G. Stevanato, L.J. Brown, J. AlonsoValdesueiro, L. Emsley, S. Jannin and M.H. Levitt, Phys. Chem. Chem. Phys. 20 (15), 9755-9759 (2018).

[58] C.R. Bowers and D.P. Weitekamp, J. Am. Chem. Soc. 109 (18), 5541-5542 (1987).

[59] R.W. Adams, J.A. Aguilar, K.D. Atkinson, M.J. Cowley, P.I.P. Elliott, S.B. Duckett, G.G.R. Green, I.G. Khazal, J. Lopez-Serrano and D.C. Williamson, Science (80-. ). 323 (5922), 1708-1711 (2009).

[60] T. Jonischkeit, U. Bommerich, J. Stadler, K. Woelk, H.G. Niessen and J. Bargon, J. Chem. Phys. 124 (20), 201109 (2006).

[61] K.V. Kovtunov, M.L. Truong, D.A. Barskiy, I.V. Koptyug, A.M. Coffey, K.W. Waddell and E.Y. Chekmenev, Chem. Eur. J. 20 (45), 14629-14532 (2014).

[62] M.B. Franzoni, L. Buljubasich, H.W. Spiess and K. Münnemann, J. Am. Chem. Soc. 134 (25), 10393-10396 (2012).

[63] D. Graafen, M.B. Franzoni, L.M. Schreiber, H.W. Spiess and K. Münnemann, J. Magn. Reson. 262, 68-72 (2016).

[64] M.B. Franzoni, D. Graafen, L. Buljubasich, L.M. Schreiber, H.W. Spiess and K. Münnemann, Phys. Chem. Chem. Phys. 15 (40), 17233-17239 (2013).

[65] Y. Zhang, P.C. Soon, A. Jerschow and J.W. Canary, Angew. Chemie - Int. Ed. 53 (13), 3396-3399 (2014).

[66] J. Eills, G. Stevanato, C. Bengs, S. Glöggler, S.J. Elliott, J. Alonso-Valdesueiro, G. Pileio and M.H. Levitt, J. Magn. Reson. 274, 163-172 (2017).

[67] B. Ripka, J. Eills, H. Kouřilová, M. Leutzsch, M.H. Levitt and K. Münnemann, Chem. Commun. 54 (86), 12246-12249 (2018).

[68] S.S. Roy, P.J. Rayner, P. Norcott, G.G. Green and S.B. Duckett, Phys. Chem. Chem. Phys. 18 (36), 24905-24911 (2016).

[69] S.S. Roy, P. Norcott, P.J. Rayner, G.G. Green and S.B. Duckett, Angew. Chemie - Int. Ed. 55 (50), 15642-15645 (2016).

[70] T. Theis, G.X. Ortiz, A.W.J. Logan, K.E. Claytor, Y. Feng, W.P. Huhn, V. Blum, S.J. Malcolmson, E.Y. Chekmenev, Q. Wang and W.S. Warren, Sci. Adv. 2 (3), e1501438 (2016).

[71] S. Glöggler, S.J. Elliott, G. Stevanato, R.C. Brown and M.H. Levitt, RSC Adv. 
7 (55), 34574-34578 (2017).

[72] I. Marco-Rius, M.C. Tayler, M.I. Kettunen, T.J. Larkin, K.N. Timm, E.M. Serrao, T.B. Rodrigues, G. Pileio, J.H. Ardenkjaer-Larsen, M.H. Levitt and K.M. Brindle, NMR Biomed. 26 (12), 1696-1704 (2013).

[73] H. Nonaka, R. Hata, T. Doura, T. Nishihara, K. Kumagai, M. Akakabe, M. Tsuda, K. Ichikawa and S. Sando, Nat. Commun. 4 (1), 2411 (2013). 
a

$\left|T_{-1}\right\rangle$

$\left|T_{0}\right\rangle-\left|S_{0}\right\rangle$ b

$|\beta \beta\rangle=$

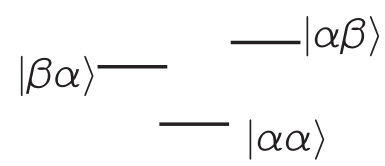

Figure 1. Energy level diagram for a a pair of magnetically equivalent spins $I=1 / 2$ and a a pair of chemically inequivalent spins $I=1 / 2$. 


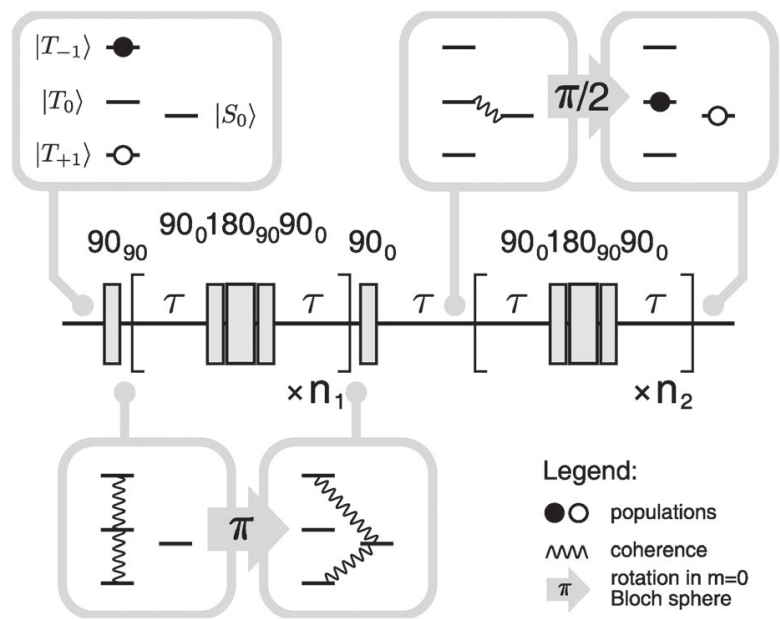

Figure 2. The 'Magnetisation-to-Singlet' (M2S) RF pulse sequence. Reproduced with permission from Refs [19] 


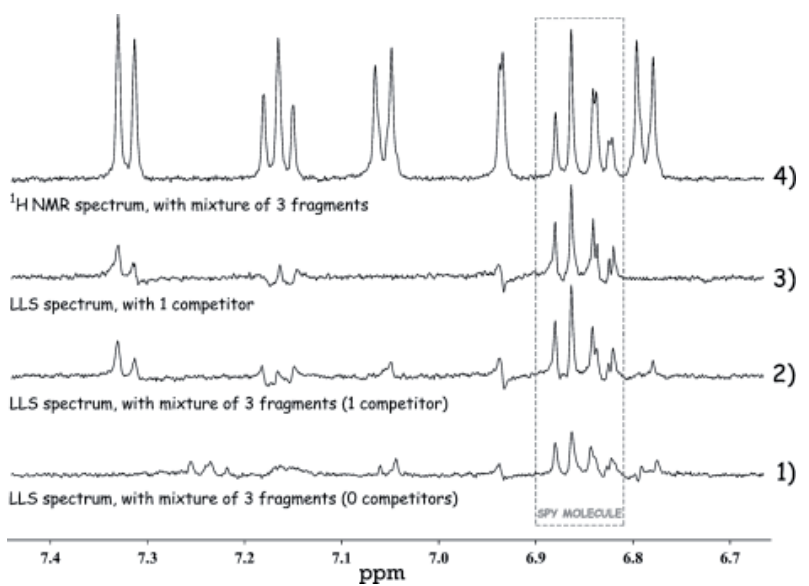

Figure 3. Identification of a weak binder in a mixture. 1) Weak LLS signals of the spy ligand after sustaining the LLS for $\Delta=2.5 \mathrm{~s}$ in the absence of a competing binder in mixture 1 (spy ligand [II] $=500 \mu \mathrm{M}$ with $K_{D}=790 \mu \mathrm{M}$ protein [Hsp90] $=2.5 \mu \mathrm{M}$ and three nonbinding ligands: $600 \mu \mathrm{M}$ tyrosine, $600 \mu \mathrm{M} 3,4-$ difluorobenzylamine, and $600 \mu \mathrm{M}$ 4-trifluoromethylbenzamidine). 2) Enhanced LLS signals in the presence of a weak binder (mixture 2 contains $600 \mu \mathrm{M}$ of the weakly binding ligand [V] 3-bromo-5-methylpyridin-2-ylamine $\left(K_{D}=2.2 \mathrm{mM}\right)$, instead of $600 \mu \mathrm{M}$ of the nonbinding ligand 3,4-difluorobenzylamine). 3) LLS signals observed in the presence of only the binding fragment (mixture 3 contains $500 \mu \mathrm{M}$ spy ligand [II], $2.5 \mu \mathrm{M}$ protein [Hsp90], and $600 \mu \mathrm{M}$ of the weakly binding ligand [V] 3-bromo-5-methylpyridin-2-ylamine). 4) Conventional ${ }^{1} \mathrm{H}$ NMR spectrum of mixture 2. Reproduced with permission from Refs [34] 


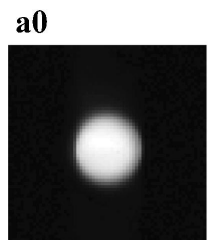

a1

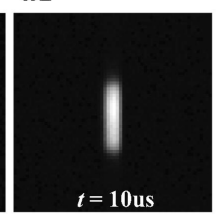

b1

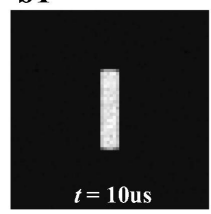

a2

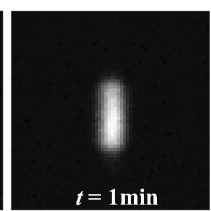

b2

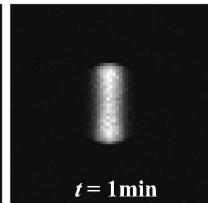

a3

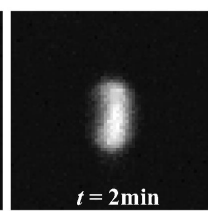

b3

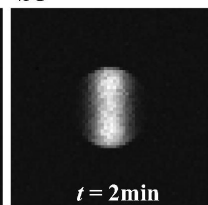

a4

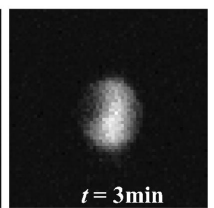

b4

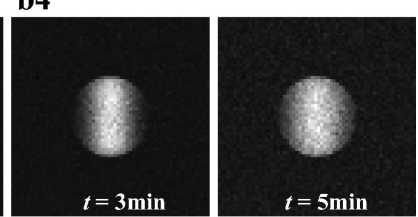

$\mathbf{a 5}$

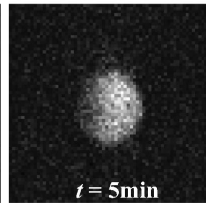

b5

Figure 4. (a) axial RARE image of a $5 \mathrm{~mm}$ OD (4.2 $\mathrm{mm}$ ID) NMR tube containing a degassed $1 \mathrm{M}$ solution of 1-(Ethyl-d 5$) 4$-(propyl-d $\left.\mathrm{d}_{7}\right)(\mathrm{Z})$-but-2-enedioate in $\mathrm{CD}_{3} \mathrm{CN}$. (a1?a5) Real space imaging of macroscopic diffusion obtained by pulse sequence in Fig. 2 of Ref. [47]. The value of the diffusion interval $\tau$ is indicated. FOV $=$ $20 \times 20 \mathrm{~mm}^{2}$, matrix size $=64 \times 64$, sagittal slice thickness $=0.9 \mathrm{~mm}$, axial slice thickness $=15 \mathrm{~mm}, \mathrm{TE}=8.7$ ms, acceleration factor 64. (b1?b5) Simulation of diffusion in a $5 \mathrm{~mm}$ OD NMR tube using a random walk model, a diffusion constant of $1.6 \times 10^{-9} \mathrm{~m}^{2} \mathrm{~s}^{-1}$ and all other parameters as used in the experiment. Reproduced with permission from Ref. [47] 


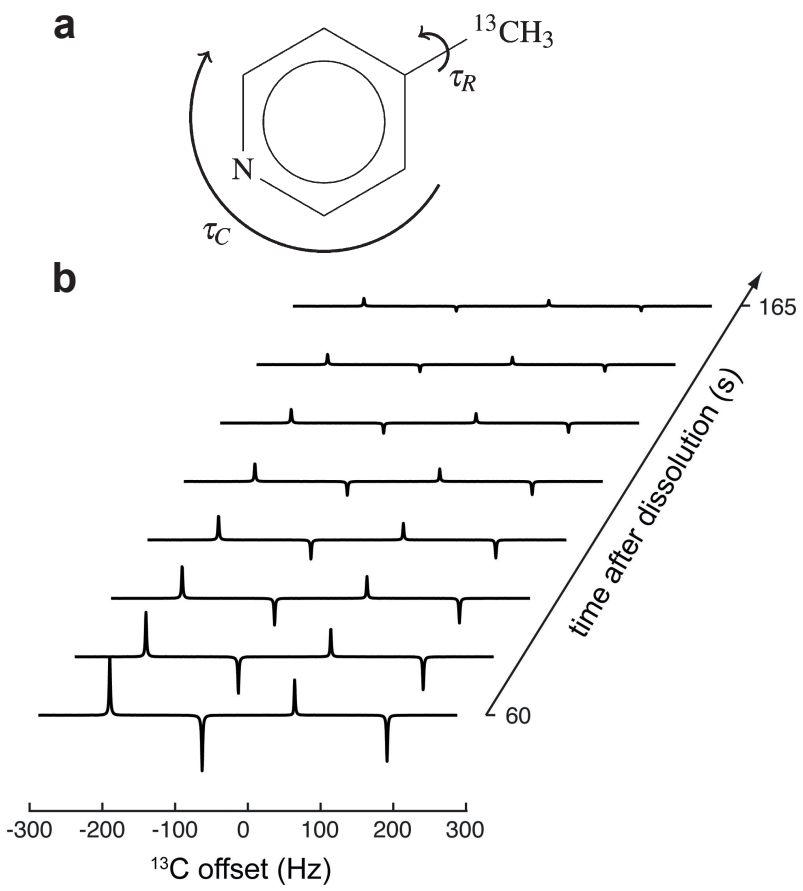

Figure 5. (a) Structure of ${ }^{13} \mathrm{C}-\gamma$-picoline. Relaxation of the LLS is controlled by correlation times $\tau_{R}$ and $\tau_{C}$ which characterise the rotational diffusion of the $\mathrm{CH}_{3}$ group and the entire molecule, respectively. (b) Selection of ${ }^{13} \mathrm{C} 1 \mathrm{D}$ spectra of the methyl signal of $2^{-13} \mathrm{C}$-acetate from the time series obtained with the experimental procedure shown in Figure 2 of Ref. [31] with positive DNP at $11.7 \mathrm{~T}$ and $298 \mathrm{~K}$. The first spectrum shown here was obtained ?60 s after dissolution. Subsequent spectra are obtained every $15 \mathrm{~s}$. Reproduced with permission from Refs $[28]$ and [31] 

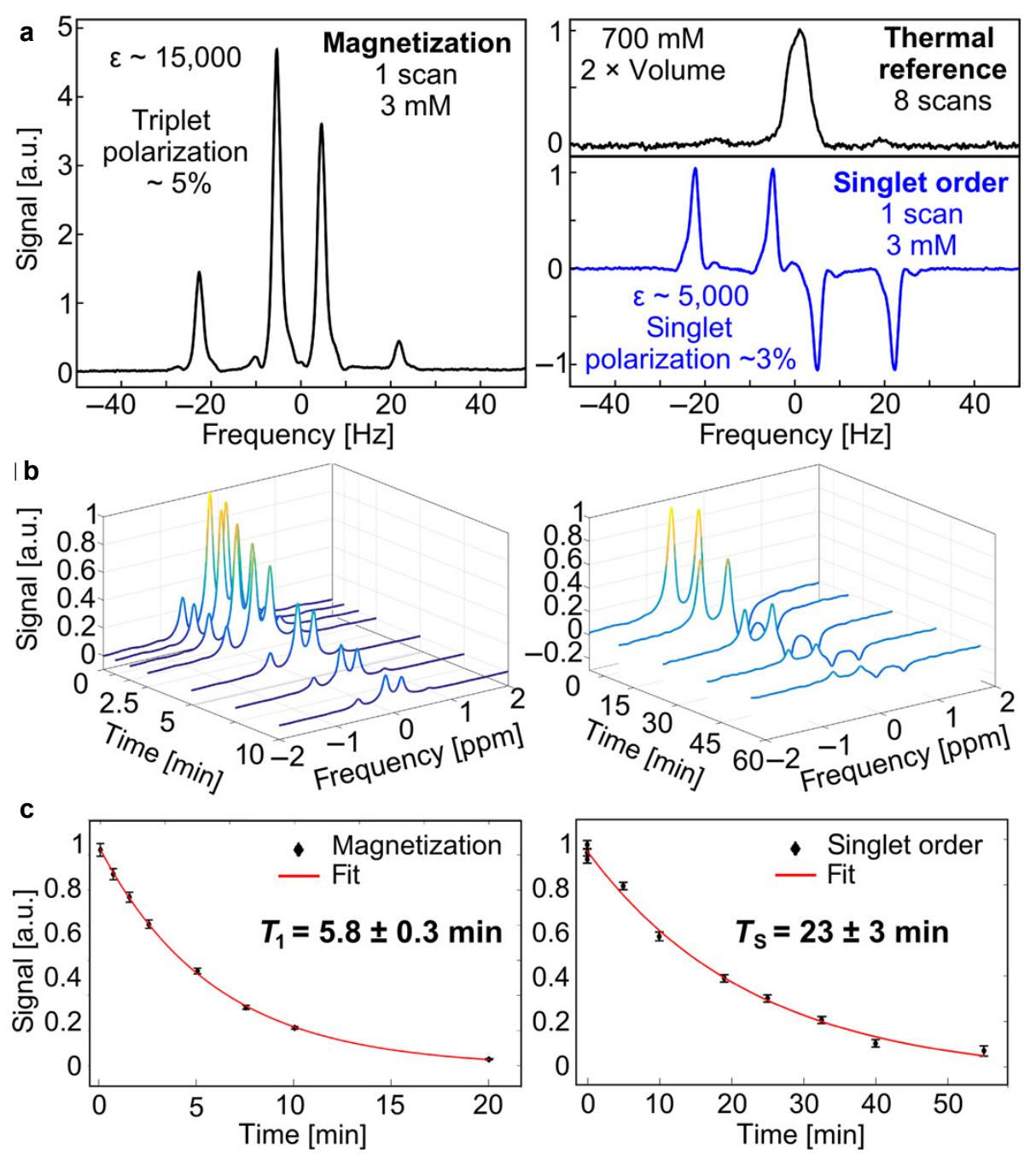

Figure 6. (a) Single-shot SABRE-SHEATH spectra obtained from a methanol- $\mathrm{d}_{4}$ solution containing 3

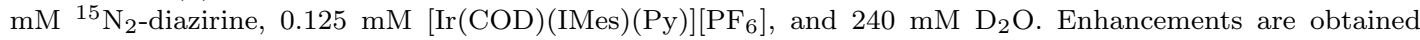
by comparison to the displayed thermal reference spectrum of the ${ }^{15} \mathrm{~N}_{2}$-diazirine [2-cyano-3- $\left(\mathrm{D}_{3}\right.$-methyl- ${ }^{15} \mathrm{~N}_{2}$ diazirine)-propanoic acid] in $\mathrm{D}_{2} \mathrm{O}$. ( $\mathrm{In}_{2} \mathrm{O}$, the chemical shift difference between the ${ }^{15} \mathrm{~N}$ nuclei is smaller than that in $\mathrm{MeOH}$, and therefore, only one peak is observed.) (b) Representative $T_{1}$ and $T_{S}$ decay measurements. $T_{1}$ is measured at $120 \mathrm{G}(12 \mathrm{mT})$ from a methanol- $\mathrm{d}_{4}$ solution containing $12 \mathrm{mM}{ }^{15} \mathrm{~N}_{2}$-diazirine, $0.125 \mathrm{mM}$ $\left[\mathrm{IrCl}(\mathrm{COD})\right.$ (IMes)], $1 \mathrm{mM}$ Py, and $960 \mathrm{mM} \mathrm{D}_{2} \mathrm{O} . T_{S}$ is measured at $3 \mathrm{G}(0.3 \mathrm{mT})$ from a solution containing 12 $\mathrm{mM}^{15} \mathrm{~N}_{2}$-diazirine, $0.05 \mathrm{mM}$ [ $\left.\mathrm{IrCl}(\mathrm{COD})(\mathrm{IMes})\right]$, and $0.4 \mathrm{mM} \mathrm{Py.} \mathrm{(c)} \mathrm{The} \mathrm{z-magnetization} \mathrm{and} \mathrm{singlet-order}$ components are extracted from the data sets in (b) and fit to a single exponential. Reproduced with permission from Ref. [70] 